\title{
Consensus and controversy among severe pancreatitis surgery guidelines: a guideline evaluation based on the Appraisal of Guidelines for Research and Evaluation II (AGREE II) tool
}

\author{
Wei Sun ${ }^{1}$, Li-Ya An ${ }^{1 \#}$, Xue-Dong Bao ${ }^{2 \#}$, Yu-Xing Qi ${ }^{1}$, Ting Yang ${ }^{1}$, Rui Li ${ }^{3}$, Su-Yun Zheng ${ }^{2}$, Da-Li Sun ${ }^{1}$ \\ ${ }^{1}$ Department of Gastrointestinal Surgery, Second Affiliated Hospital of Kunming Medical University/Second Faculty of Clinical Medicine, Kunming \\ Medical University, Kunming, China; ${ }^{2}$ Department of Digestive Endoscopy Center, Qujing First Hospital/Qujing Affiliated Hospital of Kunming \\ Medical University, Qujing, China; ${ }^{3}$ ICU, Qujing First Hospital/Qujing Affiliated Hospital of Kunming Medical University, Qujing, China \\ Contributions: (I) Conception and design: W Sun, DL Sun; (II) Administrative support: DL Sun; (III) Provision of study materials or patients: DL \\ Sun; (IV) Collection and assembly of data: LY An, XD Bao, YX Qi, T Yang; (V) Data analysis and interpretation: R Li, SY Zheng; (VI) Manuscript \\ writing: All authors; (VII) Final approval of manuscript: All authors. \\ \#These authors contributed equally to this work. \\ Correspondence to: Rui Li. ICU, Qujing First Hospital/Qujing Affiliated Hospital of Kunming Medical University, Qujing 655000, China. \\ Email: ventricular@163.com; Su-Yun Zheng. Department of Digestive Endoscopy Center, Qujing First Hospital/Qujing Affiliated Hospital of \\ Kunming Medical University, Qujing 655000, China. Email: 1227920977@qq.com; Da-Li Sun. Department of Gastrointestinal Surgery, Second \\ Affiliated Hospital of Kunming Medical University/Second Faculty of Clinical Medicine, Kunming Medical University, Kunming 650101, China. \\ Email: sundali2018@126.com.
}

\begin{abstract}
The purpose of this study was to systematically evaluate guidelines for surgery in patients with severe pancreatitis and to identify gaps limiting evidence-based medicine practice. A systematic search of databases and related websites was conducted to identify surgical guidelines for patients with severe pancreatitis. The quality of the included guidelines was assessed using the Appraisal of Guidelines for Research and Evaluation II (AGREE II) tool. The similarities among key recommendations were compared, and the evidence supporting them was extracted and analysed. Seven surgical guidelines for patients with severe pancreatitis were included. Only two guidelines, those of the World Society of Emergency Surgery (WSES) and the European Society of Gastrointestinal Endoscopy (ESGE), scored more than $60 \%$ for overall quality and were worthy of clinical recommendation. We found that the quality of the severe acute pancreatitis surgical guidelines have much room for improvement, especially in the field of application, the participation of stakeholders and editorial independence. The heterogeneity and causes of surgical recommendations were further analysed, and the latest evidence was retrieved. It was found that the surgical guidelines for severe pancreatitis lacked high-quality evidence, some of the recommendations were controversial, and evidence citation was unreasonable. The quality of surgical guidelines for patients with severe pancreatitis varies widely. In the past 5 years, the key recommendations of the surgical guidelines for severe pancreatitis have been somewhat consistent and controversial, and improvement in these existing problems and controversies will be an effective way for developers to upgrade the surgical guidelines for severe pancreatitis.
\end{abstract}

Keywords: Severe pancreatitis; surgery; guidelines; assessment

Submitted Apr 05, 2020. Accepted for publication Aug 13, 2020.

doi: 10.21037 /gs-20-444

View this article at: http://dx.doi.org/10.21037/gs-20-444 


\section{Introduction}

Severe acute pancreatitis is a serious disease of the digestive system and a dangerous condition, and it has many complications, often accompanied by organ failure. When organ failure and infectious pancreatic necrosis occur simultaneously, the condition is very serious. In a systematic review and meta-analysis including 6,970 patients (1), the mortality rate of patients with infectious pancreatic necrosis and organ failure in pancreatitis was $35.2 \%$. If patients had infectious pancreatic necrosis without organ failure, the mortality rate was $1.4 \%$.

Clinicians first try to give conservative symptomatic treatment to most patients with severe pancreatitis, and surgical intervention is the last resort to treat pancreatic infectious necrosis, especially with organ failure, as the mortality rate can increase to $25-40 \%$ in patients who undergo surgery for severe pancreatitis (2). Therefore, the selection of appropriate surgical methods, surgical timing, and surgical strategies are very important for patients with severe pancreatitis. In recent years, some organizations and experts have developed guidelines for the surgical treatment of severe pancreatitis (3-9), which aims to regulate the current severe disease. Although the contents of these guidelines are related to pancreatitis surgery, the recommendations on severe pancreatitis surgery in various guidelines are currently controversial, and the evidence is not selected properly, which is not conducive to the use of the guidelines.

Therefore, we used the Appraisal of Guidelines for Research and Evaluation II (AGREE II) tool to evaluate the methodological quality of the international guidelines for severe pancreatitis surgery in the past 5 years, and at the same time, we extracted recommendations and corresponding evidence related to severe pancreatitis surgery to evaluate the consistency and controversy among the recommendations. This review provides guidelines for guideline developers to upgrade their guidelines for severe pancreatitis surgery and helps clinicians choose the most appropriate guidelines and recommendations.

\section{Methods}

\section{Study design}

This study was conducted in accordance with Preferred Reporting Items for Systematic Reviews and MetaAnalyses (PRISMA) guidelines. We searched the English guidelines for severe pancreatitis surgery in the past 5 years (January 2015 to December 2019). The search strategy was as follows: "acute severe pancreatitis", "ASP", "acute edematous pancreatitis", "hemorrhagic necrotizing" pancreatitis", "acute biliary pancreatitis", "ABP", "surgery", “surgical treatment”, “surgical transplantation”, "statement, guideline", "recommendation", "consensus", and "diagnosis". We searched the following databases: PubMed, Web of Science, Ovid, and ScienceDirect. The search conditions were limited to titles. Manual searches of references that were included in the guidelines were performed to identify possible related studies.

\section{Data sources and literature selection process}

The inclusion criteria were as follows: (I) guidelines for surgical treatment of severe pancreatitis; (II) guidelines focused on a discussion of the best surgical indications and specific surgical methods for severe pancreatitis; (III) guidelines with full text available and published within the last 5 years; (IV) guidelines published in English; and (V) if the guidelines had been updated several times, the latest version was selected. Exclusion criteria: (I) guidelines that were repeatedly published; (II) documents that were reviews, interpretations, or summaries of relevant guidelines; (III) guidelines for which the full text was not available; and (IV) guidelines that were outdated. Two reviewers (W Sun and LY An) independently reviewed the included guidelines. The full text of the guidelines meeting the inclusion criteria was reviewed, and relevant data were extracted from the guidelines.

\section{Quality appraisal of the guidelines}

We used the AGREE II tool to evaluate the quality of guidelines. The AGREE II tool is a validated assessment tool designed to provide a framework for the evaluation and monitoring of clinical guidelines for the measurement and quantification of the quality of guidelines (10). The AGREE II tool includes 23 projects in the following 6 areas: Area 1, scope and purpose; Area 2, participants; Area 3 , rigorous formulation; Area 4, clarity of expression; Area 5, applicability; and Area 6, editorial independence. Each field was evaluated by four reviewers (W Sun, LY An, XD Bao, YX Qi), and each item was scored on a 7-point scale, in which 1 indicated strong disagreement and 7 indicated strong agreement. A score of 1 was given when little or no relevant information was provided. When the statement did not fully meet the criteria or considered only one item in the criteria, the score was 2 to 6 . The closer the 
criteria were or the more they were considered, the higher the score. A score of 7 was given when the statement met or fully considered all the criteria. All items with a score difference of 3 or more were discussed further. Finally, a reviewer summarized all the scores for each item and calculated the scores for each domain using the following formula: (acquired score - minimum possible score)/ (maximum possible score - minimum possible score)* $100 \%$. After reviewing 23 items and the comprehensive judgement of the reviewers, the evaluation guidelines were divided into three categories according to the AGREE II score, that is, recommended, revised recommended, and not recommended. The AGREE II manual does not provide guidance on how to interpret scores. To facilitate the consistency of the AGREE II tool in the evaluation of existing guidelines and recommendations for the level of evidence in all included guidelines, we adopted the following method: if the overall guideline score was $>60 \%$, it was recommended; if the overall guideline score was $30 \%$ to $60 \%$, it was recommended after modification; and if the guideline score was $<30 \%$, it was not recommended.

\section{Guidelines for the evaluation of items and evidence related to severe pancreatitis surgery}

We referred to the guidelines with relatively high AGREE II scores to extract and analyse important recommendations related to surgery for severe pancreatitis to further obtain and analyse the highest level of evidence supporting these recommendations and the highest evidence currently available in the search database. The level of recommendation was determined through the reclassification of this evidence using the Oxford Centre for Evidence-Based Medicine (OCEBM) grading system (Table S1) (11).

\section{Statistical analysis}

Each domain was calculated by using a standardized score descriptive statistical analysis and expressed as a percentage. We also listed the median and range of each domain. We used a two-way analysis of variance to calculate intra-group correlation coefficients (ICCs) to test whether the scores of the four evaluators were consistent. An ICC between 0.01 and 0.20 was considered a minor consistency, 0.21 to 0.40 fair, 0.41 to 0.60 moderate, 0.61 to 0.80 substantial, and 0.81 to 1.00 was considered very good (12). $\mathrm{P}<0.05$ was considered statistically significant. Statistical analysis was performed using the Statistical Product and Service Solutions (SPSS) version 17.0.

\section{Results}

\section{Guideline characteristics}

On the basis of the search strategy and keywords, by searching the database and website, we obtained a total of 208 search results. After removing duplicates and reading the titles, we identified 63 articles that could be further evaluated, and finally, there were 7 guidelines for severe pancreatitis surgery that met our guideline evaluation inclusion criteria (Figure 1). The basic characteristics of the seven guidelines included in this study are shown in Table 1. The included guidelines were published from 2015 to 2019. One was drafted internationally, one was drafted in Europe, and the remaining guidelines were drafted by Italy, Japan, Canada, the United States, and China. See Table 1 for other characteristics.

\section{Quality evaluation of guidelines}

The results of using the AGREE II tool to evaluate the quality of all included guidelines are shown in Table 2. The scope and purpose were $65.7 \%$ (range, 40.3-95.8\%), and the clarity of presentation was $84.7 \%$ (range, 55.6-94.4\%). Both median values were relatively high. Stakeholder involvement was $37.3 \%$ (range, 11.1-83.3\%), applicability was $25.0 \%$ (range, $0-50.0 \%$ ), and editorial independence was $48.9 \%$ (range, $0.0-95.5 \%$ ); the median values of the three were relatively low. The rigour of development was $58.7 \%$ (range, 29.7-95.3\%). Finally, we gave an overall recommendation based on the score. The overall score for each guideline is listed in Table 2. There were 2 guidelines with an overall score greater than $60 \%$, which were recommended. There were 5 guidelines with overall scores between $30 \%$ and $60 \%$; these guidelines fell into the recommended category but need to be improved. Four assessors participated in the evaluation of the surgical guidelines for severe pancreatitis. In this study, the ICCs of the AGREE II evaluations performed by the four evaluators were all greater than 0.8 , indicating that the consistency of the project scores among the evaluators was high.

\section{Recommended items and highest supporting evidence in the surgical guidelines for severe pancreatitis}

To further analyse the reasons for the heterogeneity in the recommendations for severe pancreatitis surgery in different guidelines, we referred to a high-quality guideline on major recommendations for surgery for severe pancreatitis (3), 


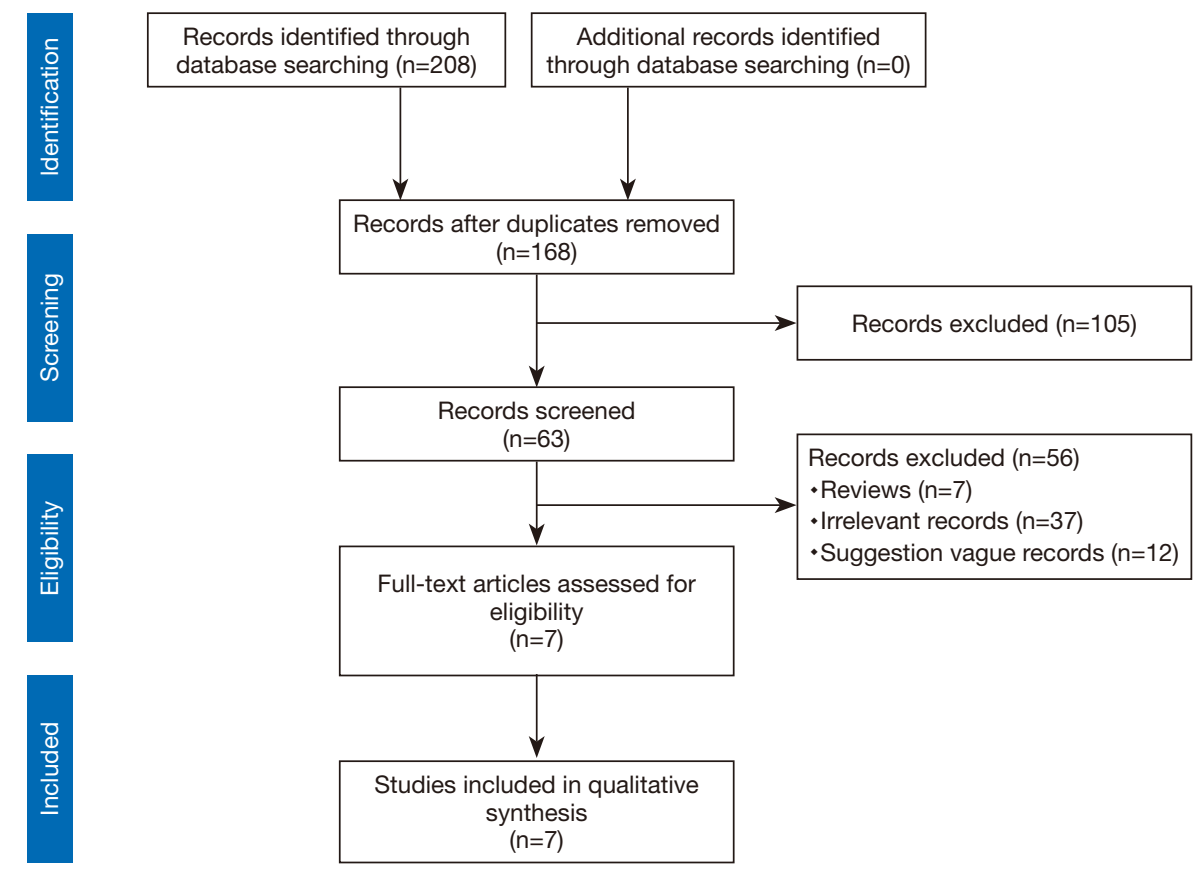

Figure 1 Flow chart of study selection.

extracted the recommendations in the guidelines and collated the highest evidence supporting these recommendations (Table 3). We included emergency endoscopic retrograde cholangiopancreatography (ERCP) indications, indications for drainage, indications for surgical intervention, the timing of surgery, surgical strategy, timing of cholecystectomy, indications for open surgery, etc. (Table 3).

\section{Discussion}

In this study, we performed a comprehensive evaluation of the surgical guidelines for severe pancreatitis using the AGREE II tool. We found that there was some consistency among the different guidelines in the recommendations for indications for emergency ERCP, drainage, surgical interventions, surgical timing, surgical strategies, cholecystectomy, and open surgery in patients with severe pancreatitis. However, these guidelines generally had unreasonable evidence references, and specific recommendations, such as the indications for surgery for infectious pancreatic necrosis and the indications for open surgery, were controversial. In addition, the quality of the evidence and the logical heterogeneity of the methods varied from guideline to guideline and even from domain to domain within the same guideline.
The recommendations among the included guidelines varied widely; therefore, we further analysed the consistencies and controversies among the current recommendations and the corresponding evidence for surgical guidelines for severe pancreatitis.

Emergency ERCP indications: acute gallstone pancreatitis with cholangitis or biliary obstruction is an indication for emergency ERCP examination (recommendation strength: $A$, level of evidence: 1 a) $(13,14)$

Although a guideline (3) also states that ERCP is not recommended for patients with severe acute gallstone pancreatitis without cholangitis or common bile duct obstruction, the authors of this recommendation believes that it is currently controversial (26), and the other included guidelines do not recommend this controversial opinion and discussion.

Indications for percutaneous or endoscopic drainage: percutaneous or endoscopic drainage can be chosen when there are signs of necrosis or pancreatitis in the clinic or if there is strong doubt (recommendation strength: $C$, level of evidence: 4) (15)

The opinions about this recommendation are more about 


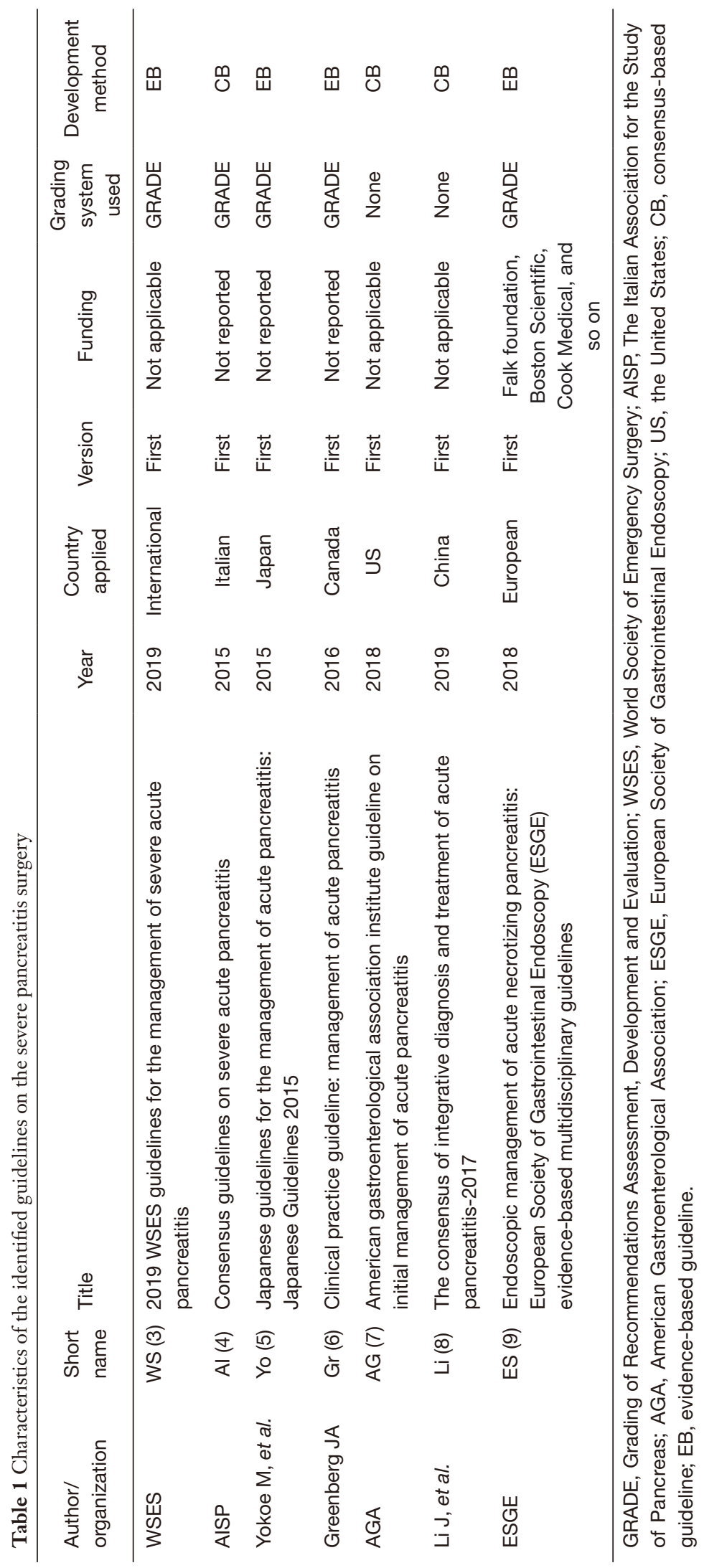


Table 2 AGREE II domain score and ICC of the included guidelines

\begin{tabular}{|c|c|c|c|c|c|c|c|c|}
\hline Guideline & $\begin{array}{l}\text { Scope and } \\
\text { purpose }\end{array}$ & $\begin{array}{l}\text { Stakeholder } \\
\text { involvement }\end{array}$ & $\begin{array}{c}\text { Rigour of } \\
\text { development }\end{array}$ & $\begin{array}{l}\text { Clarity and } \\
\text { presentation }\end{array}$ & Applicability & $\begin{array}{c}\text { Editorial } \\
\text { independence }\end{array}$ & $\begin{array}{c}\text { Overall } \\
\text { assessment }\end{array}$ & ICC \\
\hline WS (3) & $65.2 \%$ & $11.1 \%$ & $67.2 \%$ & $91.7 \%$ & $45.8 \%$ & $95.5 \%$ & $\mathrm{R}, 61.20 \%$ & 0.985 \\
\hline $\mathrm{Al}(4)$ & $50.0 \%$ & $55.5 \%$ & $67.7 \%$ & $88.9 \%$ & $0.0 \%$ & $45.8 \%$ & $\mathrm{RM}, 47.00 \%$ & 0.843 \\
\hline Yo (5) & $95.8 \%$ & $83.3 \%$ & $62.0 \%$ & $93.1 \%$ & $21.9 \%$ & $0.00 \%$ & $\mathrm{RM}, 55.00 \%$ & 0.989 \\
\hline Li (8) & $40.3 \%$ & $11.1 \%$ & $29.7 \%$ & $83.3 \%$ & $12.5 \%$ & $43.2 \%$ & $\mathrm{RM}, 32.80 \%$ & 0.986 \\
\hline ES (9) & $84.7 \%$ & $47.2 \%$ & $95.3 \%$ & $90.3 \%$ & $32.3 \%$ & $65.9 \%$ & $\mathrm{R}, 67.90 \%$ & 0.972 \\
\hline Median & $65.7 \%$ & $37.3 \%$ & $58.7 \%$ & $84.7 \%$ & $25.0 \%$ & $48.9 \%$ & - & - \\
\hline
\end{tabular}

R, recommended; RM, recommended with modifications; NR, not recommended; ICC, intra-group correlation coefficient.

understanding the natural course of the disease and the lack of high-quality randomized controlled trial (RCT) studies. The cited evidence is unreasonable; for example, a case analysis study cited in a guideline (3) addressed only surgical intervention (including drainage) and conservative treatment for clinical outcomes and found that surgical intervention can reduce mortality (27) rather than separately studying the effect of percutaneous endoscopic drainage on clinical outcomes. The evidence cited in the guideline (6) is similar, and the evidence cited is based on surgical intervention, the step-up approach and incisional necrotic tissue removal are compared, and the step-up approach includes puncture drainage (28). The guideline (7) does not give specific supporting evidence. The evidence given in a guideline (9) is to evaluate the puncture. The role of drainage in the treatment of necrotizing pancreatitis was included in a total of 11 studies, but the intervention programme was varied, and there was only one RCT.

\section{Indications for surgical intervention}

(I) In patients with abdominal compartment syndrome (ACS), an intra-abdominal pressure (IAP) $>20 \mathrm{mmHg}$ is associated with new organ dysfunction/failure (recommendation strength: C, level of evidence: 4) (16) Regarding ACS, the quality of evidence cited in the guidelines is uneven and unreasonable; for example, a guideline (8) referred to the evidence which is an abstract of a consensus meeting (29), and another guideline (9) referred to a systematic review (16). The results suggested that ACS was closely related to high mortality and complications, but the heterogeneity was obvious, the quality of the included study was low, and the results were unstable. There was a lack of research on whether surgical intervention can reduce mortality.

\section{(II) Patients with infectious pancreatic necrosis are} often accompanied by poor general condition or progressive organ failure (recommendation strength: C, level of evidence: 4) (19)

For infectious pancreatic necrosis, there was some controversy among different guidelines; for example, a guideline (4) suggested that patients with infectious pancreatic necrosis with clinically stable signs do not require surgical intervention. Imaging can indicate the formation of walled-off necrosis (WON), which can occur 4-8 weeks after the onset of pancreatitis and is an indication for endoscopic and surgical interventions. For patients with clinical signs of deterioration and ongoing necrotizing pancreatitis with organ failure, the evidence cited to support the selection of conservative management of infectious pancreatic necrosis was a meta-analysis (30). A guideline (5) considered the general deterioration of patients with infectious pancreatic necrosis as an indication for intervention, which was supported by evidence from a case-control study (31). A guideline (6) suggested that in the case of the failure of minimally invasive methods, surgical intervention should be considered, but that sufficient time is needed to delay the formation of necrotic pancreatic tissue parcels. There was no evidence to support this suggestion. 


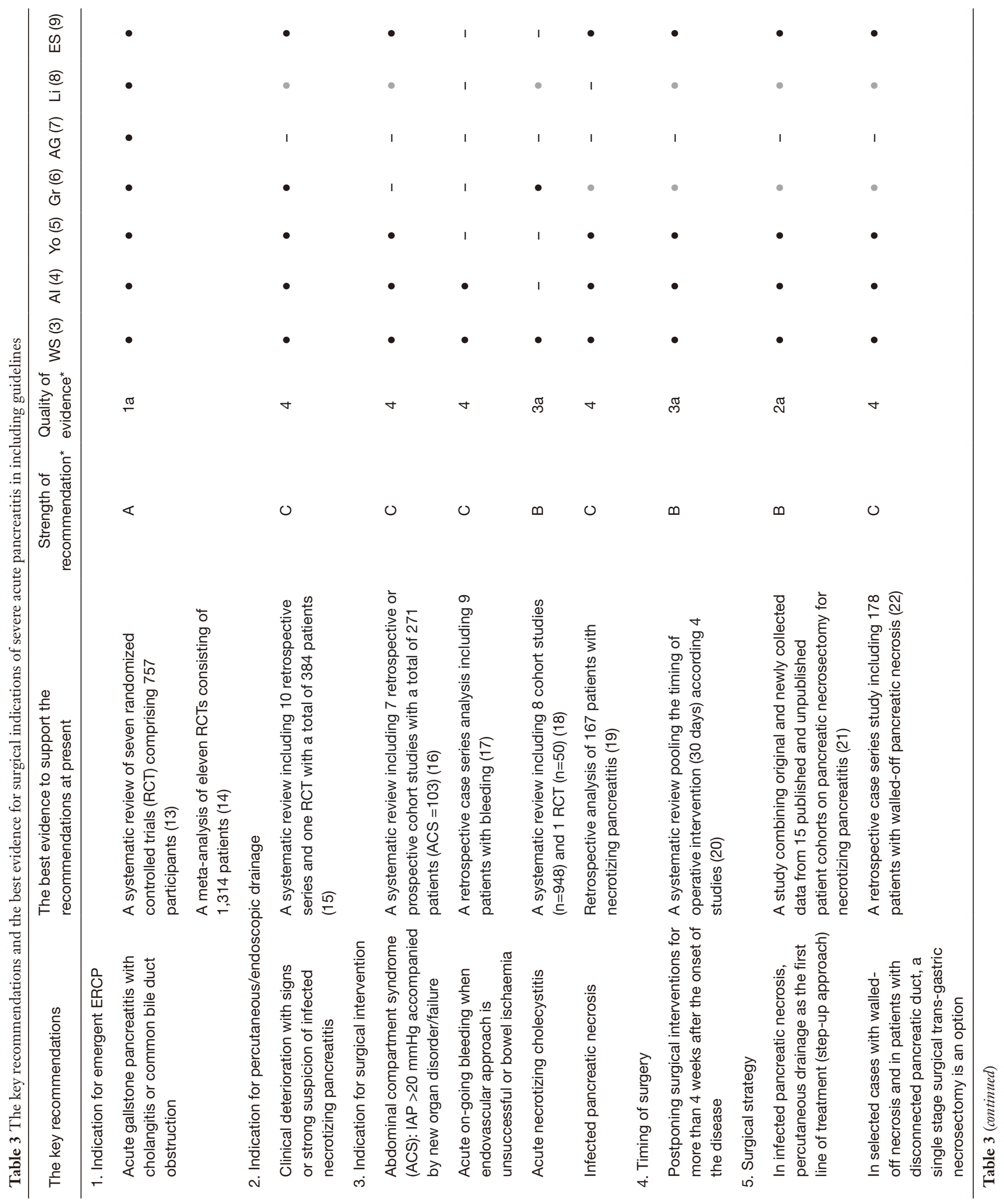


1558

Sun et al. Evaluation of SP surgery guidelines

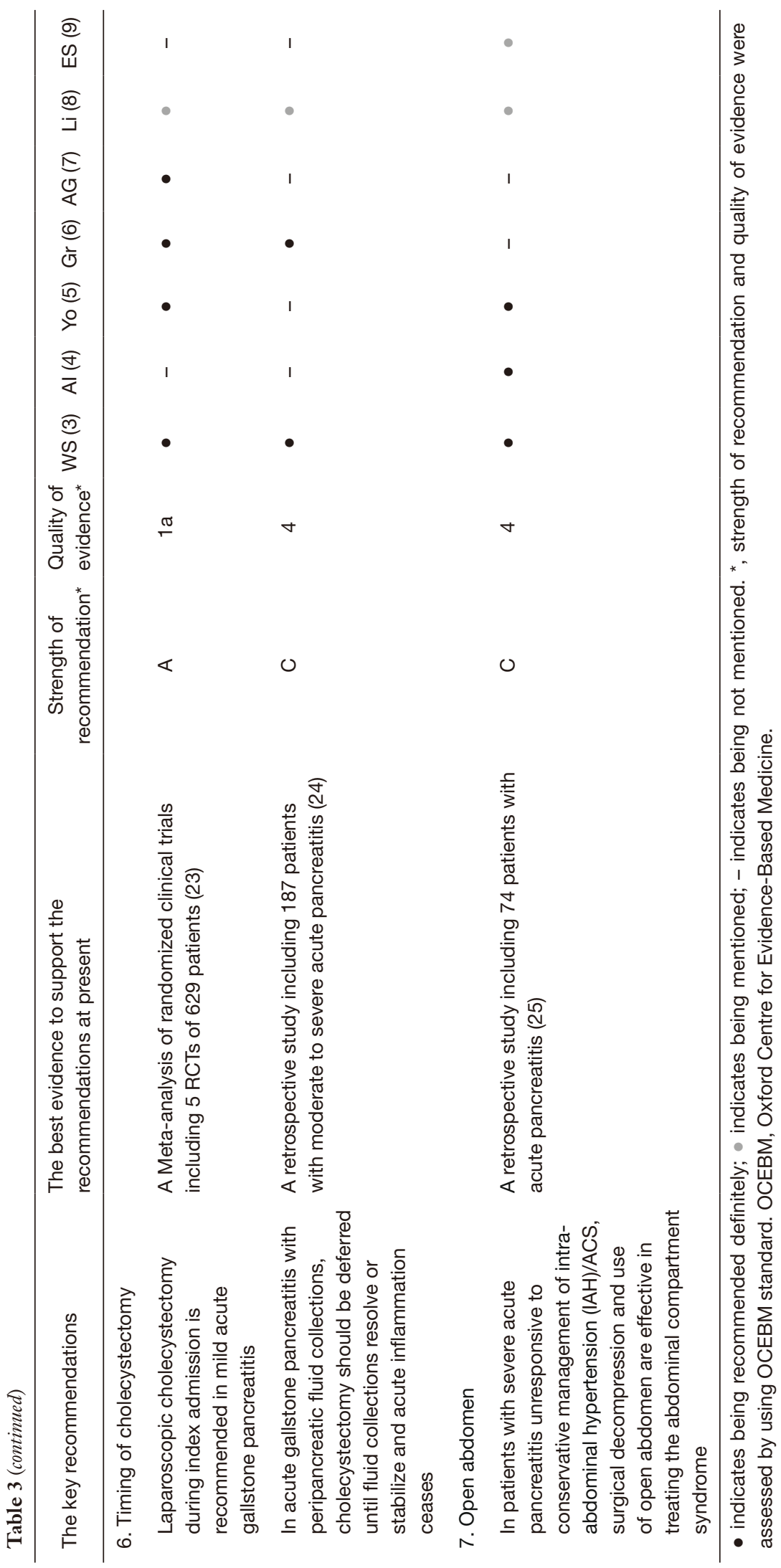

(C) Gland Surgery. All rights reserved.

Gland Surg 2020;9(5):1551-1563 | http://dx.doi.org/10.21037/gs-20-444 
Another guideline (9) suggested that confirmed infection with necrotizing pancreatitis or clinically suspected infection with necrotizing pancreatitis with progressive organ failure or persistent aggravation of general conditions should be indications for surgical intervention, and the evidence supporting this suggestion was a retrospective case analysis (19).

(III) For patients with acute necrotizing cholecystitis (recommendation strength: $\mathrm{B}$, level of evidence: $3 \mathrm{a}$ ) (18), although acute necrotizing cholecystitis is treated with more consideration for the natural course of the disease, there is a lack of high-quality research

The only evidence in guideline (6) was a systematic review. The content of the comparison was the influence of gallbladder resection and gallbladder resection on admission for acute pancreatitis recurrence (18), which does not fully support the recommendation of this article.

Surgical timing: surgical intervention should be delayed for more than 4 weeks from the onset of the disease (recommendation strength: $B$, level of evidence: $3 a$ ) (20)

The guidelines were relatively uniform on this recommendation.

\section{Surgical strategy}

(I) The step-up approach is currently recommended for the treatment of severe pancreatitis (recommendation strength: B, level of evidence: 2a) (21)

In most guidelines, recommendations were given using the highest level of evidence, mostly from the Dutch Pancreatitis Study Group's findings (28,29,32). By searching the database, we found a systematic review of the team's latest study, which included 1980 patients with necrotizing pancreatitis. It was found that minimally invasive surgery and endoscopic removal of necrotic pancreatic tissue had a lower mortality rate than open treatment (21).

(II) In patients with WON and disconnected pancreatic duct syndrome, a single-stage surgical trans-gastric necrotomy should be considered (recommendation strength: C, level of evidence: 4) (22)

There was irrationality in evidence citation. As recommended by some guidelines (4-6,9), endoscopic cleaning of necrotic tissue is superior to open operation, and the highest evidence given was a randomized controlled study (33), which does not support this recommendation. A guideline (8) recommended the removal of necrotic tissue from the transabdominal wall, but there was no evidence to support this.

\section{Timing of cholecystectomy}

(I) In patients with mild acute biliary pancreatitis, laparoscopic cholecystectomy during the same period of hospitalization is recommended (recommendation strength: A, level of evidence: 1a) (23)

The recommendation was relatively uniform among the guidelines. We retrieved the latest evidence for a metaanalysis of a randomized controlled study. It was found that for mild biliary pancreatitis, previous studies have confirmed that cholecystectomy at the same time of hospitalization can reduce the recurrence rate of pancreatitis (23).

(II) For patients with acute biliary pancreatitis with effusion around the pancreas, cholecystectomy should be delayed until the effusion subsides or stabilizes or the acute inflammatory response is stopped (recommendation strength: $C$, level of evidence: 4) (24) However, for severe pancreatitis, there is currently a lack of high-quality research evidence.

Indications of open surgery: in the case that conservative treatment of intra-abdominal bypertension (IAH)/ACS patients with severe acute pancreatitis is ineffective, surgical decompression and laparotomy are effective means in the treatment of ACS (recommendation strength: $C$, level of evidence: 4) (25)

There are different emphases on the recommendations of different guidelines for the indication of open abdominal surgery. For example, a guideline (4) suggested that in the case of ineffective puncture and drainage for ACS treatment, laparotomy can be considered, but no specific evidence was given. A guideline (5) held that for patients with an IAP $>20 \mathrm{mmHg}$, when medical treatment is ineffective and accompanied by new organ dysfunction, open abdominal drainage should be considered, citing a case-control study as the highest evidence (25). A guideline (8) suggested that in the early stage, in patients with serious ACS or persistent organ failure for more than 2 weeks that does not improve, in patients with massive ascites with obvious symptoms of infection or ascites that cannot be nonoperatively treated, in patients in the intensive care unit (ICU) for 2-3 days, 
in patients in whom there is still no improvement or even deterioration of whole-body symptoms or signs, or for those whose signs of shock or important organ dysfunction are not correct, surgical treatment should be considered. At the later stage (4 weeks later), WON, an infection of the peripancreatic or peritoneal cavity, increasing pseudocysts in the pancreas, and wrapped necrosis of the pancreas are associated with symptoms of the compression of adjacent organs. The only evidence to support these recommendations was the results of other guidelines or consensus meetings. Another guideline (9) stated that the current step-up approach has replaced the traditional direct incision of pancreatic necrotic tissue for clearance. Minimally invasive surgical removal of pancreatic necrotic tissue is not considered unless clinical symptoms are not well improved after adequate drainage. The evidence cited was from a randomized controlled study comparing the step-up approach with open abdominal surgery (28).

In summary, at present, the following problems and suggestions in the surgical guidelines for severe pancreatitis exist: (I) currently, there is good consistency among the guidelines for indications for emergency ERCP for severe pancreatitis, surgical timing, a step-up approach in surgical strategies, and laparoscopic cholecystectomy for mild acute biliary pancreatitis. The clinical recommendations are clear, and the level of reference evidence is relatively high. However, other recommendations related to surgery for severe pancreatitis lack high-quality evidence. (II) There are some controversies regarding the recommendations for ERCP not being recommended for patients who are suspected to have severe acute gallstone pancreatitis without cholangitis or choledochal obstruction, patients with infectious pancreatic necrosis as the indications of surgical intervention, and indications for open abdominal surgery for ACS. We look forward to discussing the controversial recommendations in future guideline upgrades. (III) Regarding the supporting evidence for recommendations, such as indications for percutaneous or endoscopic drainage, surgical intervention, and a surgical strategy of single-stage surgical trans-gastric gastric surgery, there are unreasonable evidence references. Corrections will be made during future guideline upgrades.

In this study, for stakeholder involvement, the median total score was $37.3 \%$. The main reason for this finding is that most of the associations that develop the guidelines ignore the involvement of both users and patients. If users and patients can be invited to participate in the development of guidelines, it can improve the applicability of the guidelines to some extent and enhance the desire of clinicians to use them.

In the development of guidelines, the rigour of development is an important component, and it is an important criterion for the determination of whether guidelines are trustworthy and whether users should adopt them. Scores of the included guidelines are variable (Table 2). The obvious problems on the rigour of development are as follows: 2 guidelines did not mention the use of a systematic approach to retrieving evidence $(7,8)$, and 2 guidelines did not clearly describe the methods used to form recommendations $(6,7)$. In addition, only 2 guidelines were externally reviewed by experts prior to publication $(4,6)$. Only one guideline (9) provided an update process.

The median score of applicability in the included guidelines was $25.0 \%$. Low scores for most guidelines in applicability were strongly associated with a lack of consideration of resources that may be required in recommended applications and a lack of standards for monitoring or auditing. Regarding applicability, only two guidelines mentioned the use of recommended advice or tools $(3,6)$.

The included guidelines scored a median of $48.9 \%$ in editorial independence. Only 3 guidelines clearly stated that funding agencies or interests had no effect on the formulation of the guidelines $(3,7,8)$. The 4 guidelines (3,7-9) documented and publicized no conflicts of interest among the members of the guideline development organization. Conflicts of interest are a common and often overlooked source of bias, and the guideline development committees should give due consideration to conflicts of interest in the guideline development process to enhance the editorial independence of the guidelines.

In summary, suggestions for improving the quality of surgical guidelines for severe pancreatitis are as follows: (I) prior to implementation, the compliance of existing guidelines with quality standards should be rigorously reviewed to make the clinical use of these guidelines more standardized. (II) Guideline developers should be familiar with guideline development standards, such as the AGREE II tool. (III) The establishment of clinical guidelines should consider the aims of different groups (including patients and the public). (IV) In addition to an explanation of the evidence in the guidelines, an objective retrieval system and a comprehensive assessment of the level of evidence should be made clear, and a regular updating mechanism for the evidence listed in the guidelines should be established. (V) The guidelines should be externally reviewed by 
experts prior to publication. (VI) As much as possible, guidelines with a higher level of evidence should be selected, and meetings should be held to develop consensus recommendations. (VII) Strict investigations of conflicts of interests among developers should be conducted, and the development process should be made transparent.

Our research has certain advantages and limitations. The advantages of this study are as follows: (I) we conducted a comprehensive analysis of the surgical recommendations and relevant evidence in the recent 5-year surgical guidelines for severe pancreatitis. We found problems in recommendations and evidence related to severe pancreatitis surgery and proposed improvement methods, which can help guideline creators and users to identify gaps in practice and guide users to choose more reliable guidelines. (II) Due to the use of appropriate weights in each area of the guideline assessment, the guidance assessment and recommendations increase the reliability of the study. The limitations of this study are as follows: (I) in this study, we evaluated only guidelines written in English, excluding those published in other languages and (II) the AGREE II tool can focus only on the method of the formulation of guidelines; it cannot assess the impact of recommendations on patient clinical outcomes.

\section{Conclusions}

The surgical guidelines for severe pancreatitis lack highquality evidence, some of the recommendations are controversial, and the evidence citation is unreasonable. At the same time, with the AGREE II tool, we found that the quality of severe acute pancreatitis surgical guidelines has much room for improvement, especially in the field of application, the participation of stakeholders and editorial independence. An effective solution to these problems will be a sensible way to improve the reliability and readability of the guidelines as they are updated and developed in the future and will enhance the use of the surgical guidelines for severe pancreatitis.

\section{Acknowledgments}

Funding: This study was supported by Yunnan Health Training Project of High Level Talents (No. H2017038) and Kunming Medical University Training Project of 100 Talents (No. 60117190466) to DLS.

\section{Footnote}

Conflicts of Interest: All authors have completed the ICMJE uniform disclosure form (available at http://dx. doi. org/10.21037/gs-20-444). The authors have no conflicts of interest to declare.

Etbical Statement: The authors are accountable for all aspects of the work in ensuring that questions related to the accuracy or integrity of any part of the work are appropriately investigated and resolved.

Open Access Statement: This is an Open Access article distributed in accordance with the Creative Commons Attribution-NonCommercial-NoDerivs 4.0 International License (CC BY-NC-ND 4.0), which permits the noncommercial replication and distribution of the article with the strict proviso that no changes or edits are made and the original work is properly cited (including links to both the formal publication through the relevant DOI and the license). See: https://creativecommons.org/licenses/by-nc-nd/4.0/.

\section{References}

1. Werge M, Novovic S, Schmidt PN, et al. Infection increases mortality in necrotizing pancreatitis: a systematic review and meta-analysis. Pancreatology 2016;16:698-707.

2. Morató O, Poves I, Ilzarbe L, et al. Minimally invasive surgery in the era of step-up approach for treatment of severe acute pancreatitis. Int J Surg 2018;51:164-9.

3. Leppäniemi A, Tolonen M, Tarasconi A, et al. 2019 WSES guidelines for the management of severe acute pancreatitis. World J Emerg Surg 2019;14:27.

4. Italian Association for the Study of the Pancreas (AISP), Pezzilli R, Zerbi A, et al. Consensus guidelines on severe acute pancreatitis. Dig Liver Dis 2015;47:532-43.

5. Yokoe M, Takada T, Mayumi T, et al. Japanese guidelines for the management of acute pancreatitis: Japanese Guidelines 2015. J Hepatobiliary Pancreat Sci 2015;22:405-32.

6. Greenberg JA, Hsu J, Bawazeer M, et al. Clinical practice guideline: management of acute pancreatitis. Can J Surg 2016;59:128-40.

7. Crockett SD, Wani S, Gardner TB, et al. American Gastroenterological Association Institute Guideline on Initial Management of Acute Pancreatitis.

Gastroenterology 2018;154:1096-101. 
8. Li J, Chen J, Tang W. The consensus of integrative diagnosis and treatment of acute pancreatitis-2017. J Evid Based Med 2019;12:76-88.

9. Arvanitakis M, Dumonceau JM, Albert J, et al. Endoscopic management of acute necrotizing pancreatitis: European Society of Gastrointestinal Endoscopy (ESGE) evidence-based multidisciplinary guidelines. Endoscopy 2018;50:524-46.

10. The AGREE Next Steps Consortium. Appraisal of guidelines for research \&evaluation II 2017. 2017. Available online: http://www.agreetrust.org

11. Pentheroudakis G, Stahel R, Hansen H, et al. Heterogeneity in cancer guidelines: should we eradicate or tolerate? Ann Oncol 2008;19:2067-78.

12. Shen WQ, Yao L, Wang XQ, et al. Quality assessment of cancer cachexia clinical practice guidelines. Cancer Treat Rev 2018;70:9-15.

13. Tse F, Yuan Y. Early routine endoscopic retrograde cholangiopancreatography strategy versus early conservative management strategy in acute gallstone pancreatitis. Cochrane Database Syst Rev 2012;39:CD009779.

14. Burstow MJ, Yunus RM, Hossain MB, et al. Meta-Analysis of Early Endoscopic Retrograde Cholangiopancreatography $(\mathrm{ERCP}) \pm$ Endoscopic Sphincterotomy (ES) Versus Conservative Management for Gallstone Pancreatitis (GSP). Surg Laparosc Endosc Percutan Tech 2015;25:185-203.

15. van Baal MC, van Santvoort HC, Bollen TL, et al. Systematic review of percutaneous catheter drainage as primary treatment for necrotizing pancreatitis. Br J Surg 2011;98:18-27.

16. van Brunschot S, Schut AJ, Bouwense SA, et al. Abdominal compartment syndrome in acute pancreatitis: a systematic review. Pancreas 2014;43:665-74.

17. Fitzpatrick J, Bhat R, Young JA. Angiographic embolization is an effective treatment of severe hemorrhage in pancreatitis. Pancreas 2014;43:436-9.

18. van Baal MC, Besselink MG, Bakker OJ, et al. Timing of cholecystectomy after mild biliary pancreatitis: a systematic review. Ann Surg 2012;25 5:860-6.

19. Rodriguez JR, Razo AO, Targarona J, et al. Debridement and closed packing for sterile or infected necrotizing pancreatitis: insights into indications and outcomes in 167 patients. Ann Surg 2008;247:294-9.

20. Mowery NT, Bruns BR, MacNew HG, et al. Surgical management of pancreatic necrosis: A practice management guideline from the Eastern Association for the Surgery of Trauma. J Trauma Acute Care Surg 2017;83:316-27.

21. van Brunschot S, Hollemans RA, Bakker OJ, et al. Minimally invasive and endoscopic versus open necrosectomy for necrotising pancreatitis: a pooled analysis of individual data for 1980 patients. Gut 2018;67:697-706.

22. Driedger M, Zyromski NJ, Visser B, et al. Surgical transgastric necrosectomy for necrotizing pancreatitis-a single-stage procedure for walled-off pancreatic necrosis. Ann Surg 2020;271:163-8.

23. Moody N, Adiamah A, Yanni F, et al. Meta-analysis of randomized clinical trials of early versus delayed cholecystectomy for mild gallstone pancreatitis. Br J Surg 2019;106:1442-51.

24. Nealon WH, Bawduniak J, Walser EM. Appropriate timing of cholecystectomy in patients who present with moderate to severe gallstone-associated acute pancreatitis with peripancreatic fluid collections. Ann Surg 2004;239:741-9; discussion 749-51.

25. Chen H, Li F, Sun JB, et al. Abdominal compartment syndrome in patients with severe acute pancreatitis in early stage. World J Gastroenterol 2008;14:3541-8.

26. van Geenen EJ, van Santvoort HC, Besselink MG, et al. Lack of consensus on the role of endoscopic retrograde cholangiography in acute biliary pancreatitis in published meta-analyses and guidelines: a systematic review. Pancreas 2013;42:774-80.

27. van Santvoort HC, Bakker OJ, Bollen TL, et al. A conservative and minimally invasive approach to necrotizing pancreatitis improves outcome. Gastroenterology 2011;141:1254-63.

28. van Santvoort HC, Besselink MG, Bakker OJ, et al. A step-up approach or open necrosectomy for necrotizing pancreatitis. N Engl J Med 2010;362:1491-502.

29. Freeman ML, Werner J, van Santvoort HC, et al. Interventions for necrotizing pancreatitis: summary of a multidisciplinary consensus conference. Pancreas 2012;41:1176-94.

30. Mouli VP, Sreenivas V, Garg PK. Efficacy of conservative treatment, without necrosectomy, for infected pancreatic necrosis: a systematic review and meta-analysis. Gastroenterology 2013;144:333-340.e2.

31. Bradley EL 3rd, Allen K. A prospective longitudinal study of observation versus surgical intervention in the management of necrotizing pancreatitis. Am J Surg 1991;161:19-24; discussion 24-5. 
32. da Costa DW, Boerma D, van Santvoort HC, et al. Staged multidisciplinary step-up management for necrotizing pancreatitis. Br J Surg 2014;101:e65-79.

33. Bakker OJ, van Santvoort HC, van Brunschot S, et al.

Cite this article as: Sun W, An LY, Bao XD, Qi YX, Yang T, Li R, Zheng SY, Sun DL. Consensus and controversy among severe pancreatitis surgery guidelines: a guideline evaluation based on the Appraisal of Guidelines for Research and Evaluation II (AGREE II) tool. Gland Surg 2020;9(5):1551-1563. doi: $10.21037 / g s-20-444$
Endoscopic transgastric vs surgical necrosectomy for infected necrotizing pancreatitis: a randomized trial. JAMA 2012;307:1053-61. 
Supplementary

Table S1 Levels of evidence and grades of the recommendations based on the Oxford Centre for Evidence-Based Medicine

\begin{tabular}{|c|c|c|}
\hline $\begin{array}{l}\text { Strength of } \\
\text { recommendation }\end{array}$ & $\begin{array}{c}\text { Quality } \\
\text { of evidence }\end{array}$ & Description \\
\hline \multirow[t]{3}{*}{ A } & $1 a$ & Systematic review (SR) with homogeneity of randomized controlled trials (RCTs) \\
\hline & $1 \mathrm{~b}$ & Individual RCT (with narrow confidence interval) \\
\hline & $1 c$ & All or none \\
\hline \multirow[t]{5}{*}{ B } & $2 a$ & SR (with homogeneity) of cohort studies \\
\hline & $2 b$ & Individual cohort study (including low-quality RCT; for example, $<80 \%$ follow-up) \\
\hline & 2c & "Outcomes" research; ecological studies \\
\hline & $3 a$ & SR with homogeneity of case-control studies \\
\hline & $3 b$ & Individual case-control study \\
\hline $\mathrm{C}$ & 4 & Case series (and poor-quality cohort and case-control studies) \\
\hline $\mathrm{D}$ & 5 & $\begin{array}{l}\text { Expert opinion without explicit critical appraisal, or based on physiology, bench research, or } \\
\text { "first principles" }\end{array}$ \\
\hline
\end{tabular}

